\title{
Use of an objective measure of articular stiffness to record changes in finger joints after intra-articular injection of corticosteroid
}

\author{
Philip S Helliwell
}

\begin{abstract}
Objective-(1) To measure objectively the subjective improvement in joint stiffness following intra-articular injections of corticosteroids, and (2) to record changes in joint stiffness in the first 24 hours after injection to look for changes in the physical properties of the joint that would be consistent with a crystal synovitis.

Methods-The study population consisted of 15 patients having 17 metacarpophalangeal joints injected as part of their routine care. Measurements were taken before injection, at 24 hours, and after one week. Outcome variables included articular stiffness, strength, joint range of movement, and subjective scores.

Results-At 24 hours, mean values for stiffness had increased (mean slope from 0.0085 to $0.0123 \mathrm{Nm}$ degree $^{-1}$; curve area from 0.1003 to 0.1555 units), but the increase was not significant. After one week a significant decrease in "elastic stiffness" had occurred (mean slope from 0.0085 to $0.0065 \mathrm{Nm} \operatorname{degree}^{-1} ; P=0.025$ ). Significant changes in grip, range of movement, and subjective scores were also found after one week (maximum grip from 75.3 to $85.9 \mathrm{~N}$; flexion/extension range from 87.0 to 102.4 degrees; pain visual analogue scale (VAS) from $50 \mathrm{~mm}$ to $12 \mathrm{~mm}$; stiffness VAS from $65 \mathrm{~mm}$ to 27 mm).

Conclusions-An early increase in joint stiffness in some patients following intra-articular corticosteroids is consistent with a transient synovitis. Symptoms of joint stiffness generally correlate with mechanical measures which provide a useful objective index of acute changes in joint pathophysiology.

(Ann Rheum Dis 1997;56:71-73)
\end{abstract}

Intra-articular corticosteroids are widely used to control synovial inflammation in inflammatory arthritis. Their beneficial action is apparent after 24-48 hours and may last for several months, depending on the preparation used. ${ }^{1}$ Adverse effects are few; Hollander has reported minor side effects in $1-2 \%$ and infrequent major adverse events (for example, joint sepsis): 19 cases in over 400000 injections. $^{2}$ However, some patients do complain of an initial deterioration in symptoms, usually within the first 12-24 hours. Hollander ascribed this to a transient crystal synovitis induced by the micro-crystalline corticosteroid preparations.
Indeed, the size of some corticosteroid preparations is very similar to that of monosodium urate crystals (for example, triamcinolone hexacetonide in Lederspan is $20-100$ microns; urate crystals are 5-20 microns). McCarty and Hogan have found evidence of crystal synovitis (as indicated by a leucocyte-rich synovial aspirate) following intrasynovial injection of corticosteroids in normal volunteers. ${ }^{3}$

Objective measures of joint stiffness are not widely used in clinical practice despite the almost universal use of "duration of early morning stiffness" as an outcome measure in clinical trails on patients with inflammatory arthritis. Early devices were cumbersome ${ }^{4}$ and later computer driven devices appeared to be unable to record the subjective increases in stiffness. ${ }^{56}$ Further work has shown, however, that providing allowance is made for finger circumference and forearm muscle bulk, comparative changes in joint stiffness can be made. $^{7}$

The aims of this study were (1) to measure objectively the subjective improvement in joint stiffness following intra-articular injections of corticosteroids, and (2) to record changes in joint stiffness in the first 24 hours after injection to look for changes in the physical properties of the joint that would be consistent with a crystal synovitis.

\section{Methods}

Inpatients with inflammatory arthritis requiring an intra-articular injection of corticosteroid into the third metacarpophalangeal (McP3) joint were invited to participate in the study. Basic demographic and disease details were obtained and the following measurements taken. Grip and pinch strength were measured with an electronic strain gauge dynamometer (MIE Medical Research, Leeds). ${ }^{8}$ Range of movement in the metacarpophalangeal joint was measured with a simple metallic angle goniometer using passive pressure to reach the end range. Subjective assessment of pain and stiffness was made using graduated plastic scales $100 \mathrm{~mm}$ long marked "no pain" (or stiffness) at one end and "worse pain ever" (or stiffness) at the other. Stiffness was measured with the Leeds microprocessor controlled arthrograph. ${ }^{9}$ This device imparts small $\left(8^{\circ}\right)$ sinusoidal movements to the relaxed metacarpophalangeal joint and measures resistance to movement with strain gauges bonded to the movement arm. Several cycles are averaged to produce a hysteresis curve from
Accepted for publication 21 October 1996
Rheumatology

Leeds I

Correspondence to: 
Summary of changes in study variables. Values are median (range)

\begin{tabular}{|c|c|c|c|c|c|}
\hline & Baseline & 24 hours & 1 week & $\begin{array}{l}\text { Paired t test } \\
\text { (baseline v } 24 h \text { ) } \\
\text { Pvalue }\end{array}$ & $\begin{array}{l}\text { Paired } t \text { test } \\
\text { (baseline v } 1 \text { week) } \\
\text { Pvalue }\end{array}$ \\
\hline Mean slope $\left(\mathrm{Nm}\right.$ degree $\left.^{-1} \times 10^{4}\right)$ & $85(16$ to 218$)$ & $123(21$ to 221$)$ & 65 (10 to 234$)$ & NS & 0.025 \\
\hline Area (units $\times 10^{4}$ ) & 1003 (149 to 3128$)$ & 1555 (339 to 2535$)$ & 917 (201 to 2990$)$ & NS & NS \\
\hline Maximum grip (N) & $71(15$ to 114$)$ & $77(19$ to 135$)$ & $80(19$ to 146$)$ & NS & 0.025 \\
\hline Maximum pinch $(\mathrm{N})$ & $31(10$ to 54$)$ & $34(13$ to 75$)$ & $45(13$ to 75$)$ & NS & NS \\
\hline Flexion/extension (deg) & 86.5 (71 to 99$)$ & t & $97(87$ to 124$)$ & & 0.005 \\
\hline Abduction/adduction (deg) & $32(23$ to 63$)$ & $t$ & $36(28$ to 570$)$ & & NS \\
\hline Stiffness (VAS, mm) & $65(0$ to 100$)$ & $44(0$ to 92$)$ & $27(0$ to 60$)$ & $\mathrm{NS}^{\star}$ & $0.008^{\star}$ \\
\hline Pain (VAS, mm) & $50(20$ to 100$)$ & $46(0$ to 76$)$ & $12(0$ to 76$)$ & NS* & $0.003^{\star}$ \\
\hline
\end{tabular}

ॠ Mann-Whitney test.

† Measurements not taken

VAS, visual analogue scale.

which measures of joint stiffness can be obtained: "elasticity" represents the recoverable energy of deformation (modelled as a rubber band) and "viscosity" the non-recoverable energy (modelled as a piston in a cylinder of viscous fluid). All measurements were made immediately before joint injection, at 24 hours postinjection, and at one week postinjection. In one case more frequent measurements were made over the first 24 hours after injection.

Injections into the $\mathrm{McP} 3$ joint were made by the author using the dorsal approach with the finger in light traction. All cases were injected with triamcinolone hexacetonide. The maximum volume injected did not exceed $0.5 \mathrm{ml}$. Other treatments such as physiotherapy were not applied to the injected hand during the study.

\section{Results}

Seventeen metacarpophalangeal joints were injected in 15 patients (mean age, 61 years; 10 females, five males). The majority of patients had rheumatoid arthritis, two had psoriatic arthritis. The mean disease duration was 14.1 years (range 0.5 to 36 years), mean Ritchie articular index 16.7 (6 to 28), and mean Larsen score for hands was 64.2 (24 to 113).

The results are summarised in the table. Overall there was a trend for stiffness variables to deteriorate 24 hours after injection but to improve by the end of the week. Significant changes in elastic stiffness, maximum grip,

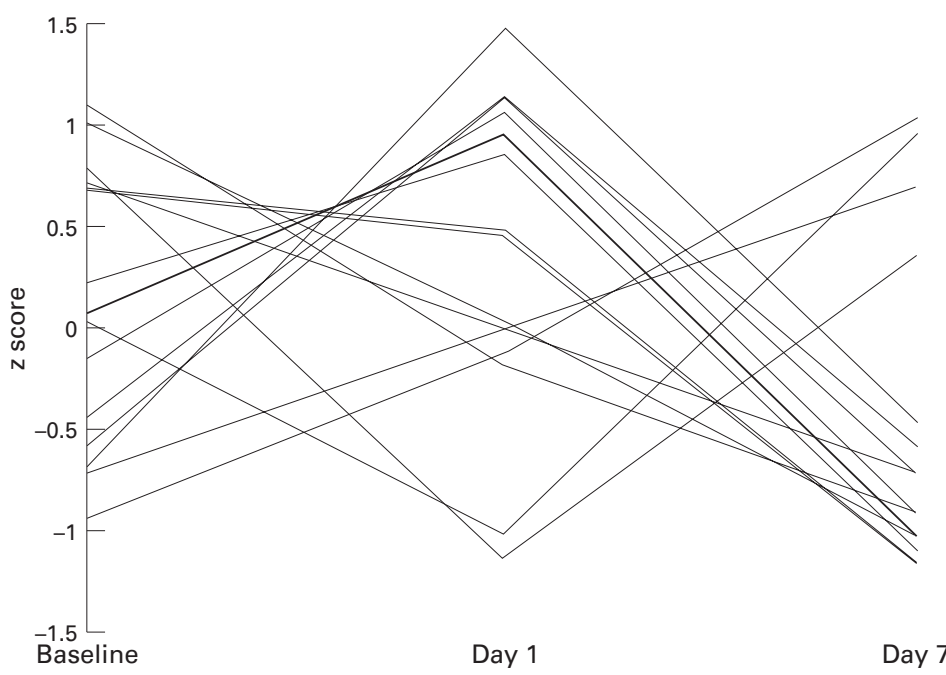

Individual changes in elastic stiffness (see text for explanation of $z$ score). range of movement, and subjective scores were seen between baseline and 1 week.

Individual changes in elastic stiffness, expressed as the standard normal deviate $[\mathrm{z}=$ $\left.\left(\mathrm{x}_{\mathrm{i}}-\overline{\mathrm{x}}\right) / \mathrm{s}\right]$ are depicted graphically in the figure. Eight patients deteriorated objectively at 24 hours (subjectively only two patients complained of a worsening of pain and four patients complained of a deterioration in stiffness). After seven days, 12 joints had improved, four had deteriorated, and one was unchanged (after being worse at 24 hours). The two groups of patients (those who deteriorated and those who improved at 24 hours) were similar with respect to age, duration of disease, Ritchie articular index, Larsen score, range of movement, and joint stiffness, but differed with respect to erythrocyte sedimentation rate (deteriorate $v$ improve: mean ESR $21 v 41$, $t=-2.55, \mathrm{P}=0.03)$.

In one patient recordings were made at four hourly intervals for the first 24 hours. No increase in stiffness was seen at four hours but by eight hours an increase had occurred.

\section{Discussion}

Intra-articular steroids are given to suppress inflammation locally. The reduction of inflammatory mediators results in less periarticular oedema, less joint effusion, and less pain, and there is a corresponding improvement in mechanical symptoms such as range of movement and stiffness. A device such as the arthrograph permits an objective assessment of the symptom of stiffness and allows serial measurements of the mechanical properties of a joint in response to therapeutic interventions such as intra-articular steroid injections. The arthrograph may be of more limited use in recording serial changes in joint stiffness over longer periods of time (years) since the absolute measure of stiffness depends on a number of factors including the degree of joint damage $^{5}$ and associated muscle wasting. ${ }^{7}$ However, in situations where joint stiffness is changing rapidly due to a reduction in local inflammation in response to treatment, the arthrograph can provide a truly objective measure of joint mechanics. In this study the expected decrease in joint stiffness was found one week after injection and this was accompanied by a corresponding decrease in subjective stiffness and pain.

However, an increase in joint stiffness at 24 hours occurred in eight joints (eight patients). Half the patients were unaware of 
this deterioration. Several issues occur here. Firstly, do subjective and objective assessments not match because they are measuring different things? The engineers' definition of stiffness (an increase in resistance to movement) matches that of the physician and has recently been shown to match that of the patient. ${ }^{10}$ Secondly, do patients perhaps tell us only what they think we want to hear? Thus in the face of a worsening (or at least a non-improvement) in their symptoms at 24 hours are they reluctant to admit this to the physician when improvement should be occurring? Thirdly, there may be a threshold effect so that a certain percentage change in stiffness must occur before it will be appreciated: of the patients who felt a deterioration, the mean increase in joint stiffness was $46.9 \%$, compared to $17.4 \%$ in the patients who did not feel this change. Fourthly, people may differ in their sensitivity to changes in stiffness, as with others forms of sensation.

The volume of fluid injected at the time of intra-articular treatment is limited by the size of the joint. For the metacarpophalangeal joint the maximum injectable volume is $0.5-1 \mathrm{ml}$; using these volumes the joint capsule will be distended and patients report this feeling of distension and increased stiffness immediately after joint injection. However, this quickly subsides. In order to avoid any effect of joint distension associated with intra-articular injections, stiffness measurements were made 24 hours after injection. In the patient in whom more frequent early measurements were made no change was seen at four hours but by eight hours an increase had occurred. In this patient at least this was not simply due to an increase in intracapsular volume.

Hollander suggested that some degree of crystal synovitis is universal following intraarticular injection of crystalline steroids. ${ }^{2}$ This would accord with what is known of the particle size of these preparations compared to, for example, uric acid crystals, although in practice it would be difficult to distinguish between crystal synovitis and chemical synovitis resulting from preservatives in the steroid preparation. Uric acid crystals are avidly phagocytosed (with subsequent cell death) when they occur free in synovial fluid and it seems likely that the same process may occur with steroid crystals. The subsequent cell rupture and release of lysosomal enzymes is responsible for the acute synovitis of gout but with steroid crystals the profound antiinflammatory effect of the steroid preparation probably prevents a marked deterioration in the synovitis and the appearance of overt inflammation in the injected joint. Nevertheless, the results of this study suggest that in some patients there may be a brief increase in joint inflammation following intra-articular steroids: this may be due to the crystalline nature of the preparation or to chemical preservatives contained therein.

I thank the Arthritis and Rheumatism Council for financial support during this study.

1 Blyth T, Hunter JA, Stirling A. Pain relief in the rheumatoid knee after steroid injection a single-blind comparison of hydrocortisone succinate, and triamcinolone acetonide or hexacetonide. Br J Rheumatol 1994;33:461-3.

2 Hollander JL. Arthrocentesis technique and intra-synovial therapy. In: McCarty DJ. Arthritis and allied conditions, 10th ed. Philadelphia: Lea and Febiger, 1987.

3 McCarty DJ, Hogan JM. Inflammatory reaction after intrasynovial injection of microcrystalline adrenocorticosteroid esters. Arthritis Rheum 1964;7:359-67.

4 Wright V, Johns RJ. Physical factors concerned with the stiffness of normal and diseased joints. Bull Johns Hopkins Hosp 1960;106:215-31.

5 Helliwell PS, Howe A, Wright V. Lack of objective evidence of stiffness in rheumatoid arthritis. Ann Rheum Dis 1988; 47:754-58.

6 Yung P, Unsworth A, Haslock I. Measurement of stiffness in the metacarpophalangeal joint: circadian variation. Clin the metacarpophalangeal joint: circa

7 Helliwell PS, Smeathers JE, Wright V. The contribution of different tissues to stiffness in the joint. Proc Inst Mech Eng, part H (Eng Med) 1995;208:223-8.

8 Helliwell PS, Howe A, Wright V. Functional assessment of the hand: reproducibility, acceptability, and utility of a new system for measuring strength. Ann Rheum Dis 1987; 46:203-8.

9 Howe A, Thompson D, Wright V. Reference values for metacarpophalangeal joint stiffness in normals. Ann Rheum Dis $1985 ; 44: 469-76$.

10 Helliwell PS. The semeiology of arthritis: discriminating between patients on the basis of their symptoms. Ann Rheum Dis 1995;54:924-6. 